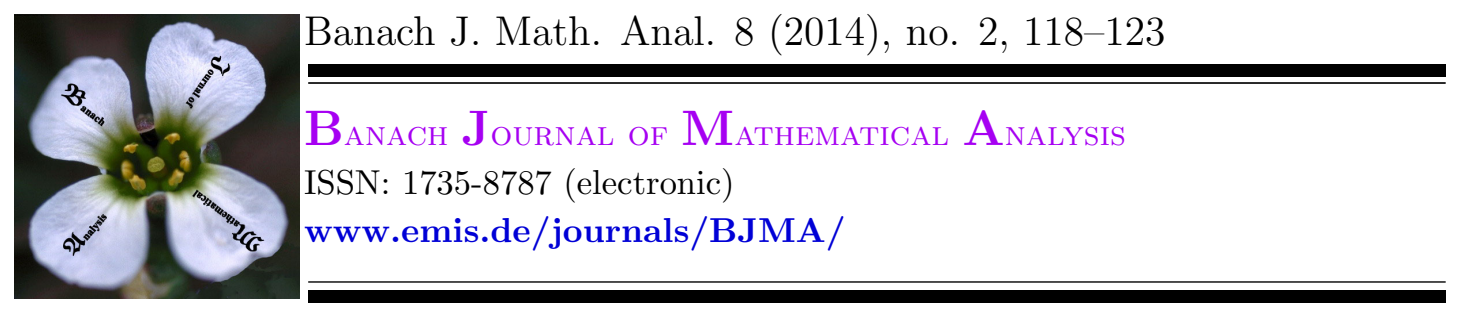

\title{
A CHARACTERIZATION OF CONVEX FUNCTIONS AND ITS APPLICATION TO OPERATOR MONOTONE FUNCTIONS
}

\author{
MASATOSHI FUJII ${ }^{*}$, YOUNG OK KIM ${ }^{2}$ AND RITSUO NAKAMOTO ${ }^{3}$ \\ Communicated by M. S. Moslehian
}

\begin{abstract}
We give a characterization of convex functions in terms of difference among values of a function. As an application, we propose an estimation of operator monotone functions: If $A>B \geq 0$ and $f$ is operator monotone on $(0, \infty)$, then $f(A)-f(B) \geq f(\|B\|+\epsilon)-f(\|B\|)>0$, where $\epsilon=\left\|(A-B)^{-1}\right\|^{-1}$. Moreover it gives a simple proof to Furuta's theorem: If $\log A>\log B$ for $A, B>0$ and $f$ is operator monotone on $(0, \infty)$, then there exists a $\beta>0$ such that $f\left(A^{\alpha}\right)>f\left(B^{\alpha}\right)$ for all $0<\alpha \leq \beta$.
\end{abstract}

\section{INTRODUCTION}

For a twice differentiable real-valued function $f$, its convexity is characterized by $f^{\prime \prime} \geq 0$. Since there are many non-differentiable convex functions, we consider a characterization of general convex functions. We cannot use the differentiation, but the average rate of change is available. Roughly speaking, we claim that the convexity of a function is characterized by the non-decreasingness of average rate of change. It seems to be natural as a generalization of the condition $f^{\prime \prime} \geq 0$. Actually it will be formulated as Lemma 1 in the next section.

To explain operator monotone functions, we introduce the operator order $A \geq$ $B$ among selfadjoint operators $A, B$ on a Hilbert space $H$ by $(A x, x) \geq(B x, x)$ for all $x \in H$. In particular, $A$ is positive if $A \geq 0$, i.e., $(A x, x) \geq 0$ for all $x \in H$.

Date: Received: Sep. 15, 2013; Accepted: Oct. 7, 2013.

* Corresponding author.

2010 Mathematics Subject Classification. Primary 47A63; Secondary 47B10, 47BA30.

Key words and phrases. Convex function, operator monotone function, Lówner-Heinz inequality, chaotic order. 
Next, a positive operator $A$ is said to be strictly positive, denoted by $A>0$, if $A \geq c$ for some constant $c>0$. So $A>B$ means that $A-B>0$.

A real-valued continuous function $f$ defined on $[0, \infty)$ is called operator monotone if it preserves the operator order, i.e., $f(A) \geq f(B)$ for $A \geq B \geq 0$. One of the most important examples is the power function $t \mapsto t^{p}$ for $0 \leq p \leq 1$ (Löwner-Heinz inequality). In general, $f$ is called operator monotone on an interval $J$ if $f(A) \geq f(B)$ for $A \geq B$ whose spectra contained in $J$. For this, we pose $\log t$ as a fundamental example of an operator monotone function on $(0, \infty)$.

Very recently, Moslehian and Najafi [9] proposed an excellent extension of the Löwner-Heinz inequality as follows:

Theorem MN. If $A>B \geq 0$ and $0<r \leq 1$, then $A^{r}-B^{r} \geq\|A\|^{r}-(\|A\|-$ $\epsilon)^{r}>0$, and $\log A-\log B \geq \log \|A\|-\log (\|A\|-\epsilon)>0$, where $\epsilon=\left\|(A-B)^{-1}\right\|^{-1}$.

In this note, we apply our characterization of concave functions and give an improvement and a generalization of Theorem MN (Theorem 5). As another application, we can give a short proof to a recent result due to Furuta [6, Theorem 2.1 , which is an operator inequality related to operator monotone functions and chaotic order, i.e., the order defined by $\log A \geq \log B$ among positive invertible operators.

\section{A CHARACTERIZATION OF CONVEX FUnCTIONS}

In this section, we propose an elementary characterization of convex functions. We essentially use average rate of change.

Lemma 2.1. A real valued continuous function $f$ on an interval $J=[a, b)$ with $b \in(-\infty,+\infty]$ is convex (resp. concave) if and only if, for each $0<\epsilon<b-a$, $D_{\epsilon}(t)=f(t+\epsilon)-f(t)$ is non-decreasing (resp. non-increasing) on $[a, b-\epsilon)$.

Proof. Suppose that $f$ is convex on $J$. Take $s, t \in J$ with $s<t$ and $t+\epsilon \in J$. We may assume that $t-s<\epsilon$. Let $y=L(t)$ be the linear function through $(s, f(s))$ and $(s+\epsilon, f(s+\epsilon))$. Then we have

$$
L(t) \geq f(t) \text { and } L(t+\epsilon) \leq f(t+\epsilon)
$$

by the convexity of $f$. Hence it implies that

$$
\begin{aligned}
D_{\epsilon}(t) & =f(t+\epsilon)-f(t) \\
& \geq L(t+\epsilon)-L(t) \\
& =L(s+\epsilon)-L(s) \quad \text { by the linearity of } \mathrm{L} \\
& =f(s+\epsilon)-f(s) \\
& =D_{\epsilon}(s)
\end{aligned}
$$

as desired.

Conversely suppose that $D_{\epsilon}(t)$ is non-decreasing. Take $t, s \in J$ with $s<t=$ $s+2 \epsilon$. Since $D_{\epsilon}(s) \leq D_{\epsilon}(s+\epsilon)$, we have

$$
2 f\left(\frac{s+t}{2}\right)=2 f(s+\epsilon) \leq f(s+2 \epsilon)+f(s)=f(t)+f(s) .
$$

So $f$ is convex. 
Corollary 2.2. If $f$ is strictly increasing and concave on an interval $[a, b+\delta]$ in $\mathbb{R}$ for some $\delta>0$, then for each $0<\epsilon \leq \delta, D_{\epsilon}(t) \geq D_{\epsilon}(b)>0$ for all $t \in[a, b]$.

Remark 2.3. Analogous argument on convexity of functions as above has been done in [8, page 2].

\section{Applications to Operator monotone functions}

As an application of Corollary 2.2, we give an estimation of operator monotone functions.

Lemma 3.1. If $f$ is non-constant and operator monotone on the interval $\mathbb{R}_{+}=$ $[0, \infty)$, then $f$ is strictly increasing.

Proof. First of all, we note that $f$ is non-decreasing. Next we suppose that $f^{\prime}(c)=0$ for some $c>0$. Noting that the Löwner matrix

$$
\left(\begin{array}{cc}
f^{\prime}(c) & f^{[1]}(c, d) \\
f^{[1]}(d, c) & f^{\prime}(d)
\end{array}\right)
$$

is positive semidefinite for any $d>0$ by the operator monotonicity of $f$, where $f^{[1]}(c, d)=\frac{f(c)-f(d)}{c-d}$ is the devided difference.

Therefore its determinant is nonnegative, so that $f^{[1]}(c, d)=0$ for any $d>0$. This means that $f$ is constant, which is a contradiction. Consequently we have $f^{\prime}>0$.

Lemma 3.2. If $C \geq 0$ and $f$ is a concave and strictly increasing function on an interval $[a, d)$ containing the spectrum of $C$, then for each $0<\epsilon<d-\|C\|$, $f(C+\epsilon) \geq f(C)+D_{\epsilon}(\|C\|)$.

Proof. We first note that for a given $0<\epsilon<d-\|C\|$, we can take $c>0$ satisfying $0<c<d$ and $\epsilon<c-\|C\|$. Applying Corollary 2.2 to $b=\|C\|$ and $\delta=c-\|C\|$, it follows that

$$
f(C+\epsilon)-f(C) \geq D_{\epsilon}(\|C\|)
$$

We here give a precise estimation of [6, Theorem 2.1] and [8, Proposition 2.2], cf. [9].

Theorem 3.3. If $A>B \geq 0$ and $f$ is non-constant operator monotone on $[0, \infty)$, then $f(A)-f(B) \geq f(\|B\|+\epsilon)-f(\|B\|)>0$, where $\epsilon=\left\|(A-B)^{-1}\right\|^{-1}$.

Proof. Since $A \geq B+\epsilon$ for $\epsilon=\left\|(A-B)^{-1}\right\|^{-1}>0$, we have

$$
f(A) \geq f(B+\epsilon) \text {. }
$$

Furthermore Lemmas 3.1 and 3.2 imply that

$$
f(B+\epsilon) \geq f(B)+D_{\epsilon}(\|B\|) .
$$

Hence we have

$$
f(A)-f(B) \geq D_{\epsilon}(\|B\|)=f(\|B\|+\epsilon)-f(\|B\|)>0 .
$$


As a consequence, we have an improvement of the estimation due to Moslehian and Najafi [9]:

Corollary 3.4. If $A>B \geq 0$ and $0<r \leq 1$, then $A^{r}-B^{r} \geq(\|B\|+\epsilon)^{r}-$ $(\|B\|)^{r}>0$, and $\log A-\log B \geq \log (\|B\|+\epsilon)-\log \|B\|>0$, where $\epsilon=\|(A-$ $B)^{-1} \|^{-1}$.

Remark 3.5. We note that Corollary 3.4 actually improves Theorem MN. Since $\|A\|-(\|A\|-\epsilon)=\epsilon=(\|B\|+\epsilon)-\|B\|$ and the function $t \mapsto t^{r}$ is strictly concave, it follows that

$$
\|A\|^{r}-(\|A\|-\epsilon)^{r} \leq(\|B\|+\epsilon)^{r}-\|B\|^{r} .
$$

We here pose an example:

$$
A=\left(\begin{array}{ll}
4 & 0 \\
0 & 2
\end{array}\right) \text { and } B=\left(\begin{array}{ll}
2 & 0 \\
0 & 1
\end{array}\right) .
$$

Then $A-B=\left(\begin{array}{ll}2 & 0 \\ 0 & 1\end{array}\right) \geq 1$ and so $\epsilon=1$. Hence we have

$$
\|A\|^{r}-(\|A\|-\epsilon)^{r}=4^{r}-3^{r}<(\|B\|+\epsilon)^{r}-\|B\|^{r}=3^{r}-2^{r} .
$$

Now Theorem 3.3 can be regarded as a difference version. So we give a ratio version of it. It is obtained by Theorem 3.3 itself:

Corollary 3.6. If $A>B>0$ and $f$ is non-constant operator monotone on $(0, \infty)$, then

$$
f(B)^{-\frac{1}{2}} f(A) f(B)^{-\frac{1}{2}} \geq 1+(f(\|B\|+\epsilon)-f(\|B\|))\|f(B)\|^{-1},
$$

where $\epsilon=\left\|(A-B)^{-1}\right\|^{-1}$.

Proof. Put $\delta=f(\|B\|+\epsilon)-f(\|B\|)$. It follows from Theorem 3.3 that

$$
\begin{gathered}
f(B)^{-\frac{1}{2}} f(A) f(B)^{-\frac{1}{2}} \geq f(B)^{-\frac{1}{2}} f(B+\delta) f(B)^{-\frac{1}{2}} \\
=1+\delta f(B)^{-1} \geq 1+\delta\|f(B)\|^{-1} .
\end{gathered}
$$

As another application of Theorem 3.3, we need the chaotic order: For $A>0$, we can define the selfadjoint operator $\log A$. So a weaker order than the operator order appears by $\log A \geq \log B$ for $A, B>0$. We call it the chaotic order. The chaotic order plays an substantial role in operator inequalities. Among others, it brightens the Furuta inequality [5], [2], [3], [1], [4], [7] and recent development of Karcher mean theory [11].

Now we give a simple and elementary proof to the following recent theorem $[6$, Theorem 2.1] due to Furuta, in which we don't use any integral representation of operator monotone functions.

Theorem 3.7. If $\log A>\log B$ for $A, B>0$ and $f$ is operator monotone on $(0, \infty)$, then there exists $\beta>0$ such that

$$
f\left(A^{\alpha}\right)>f\left(B^{\alpha}\right) \text { for all } 0<\alpha \leq \beta .
$$


Proof. Since $\log A>\log B$, it is known that there exists $\beta>0$ such that

$$
A^{\alpha}>B^{\alpha} \text { for all } 0<\alpha \leq \beta \text {. }
$$

Therefore it follows from Theorem 3.3 that, for each fixed $\alpha \in(0, \beta]$,

$$
f\left(A^{\alpha}\right)>f\left(B^{\alpha}\right)
$$

as desired.

\section{A CONCLUDING REMARK.}

Finally we discuss an operator extension of Lemma 2.1. Namely we may expect the following conjecture:

$A$ real valued function $f$ on an interval $J=(a, b)$ with $b \in(-\infty,+\infty]$ is operator convex if and only if, for each $0<\epsilon<b-a, D_{\epsilon}(t)$ is operator monotone on $(a, b-\epsilon)$. Unfortunately we have a negative answer as follows: We choose the function $f(t)=\frac{1}{t}$ on $(0, \infty)$. It is a typical example of operator convex functions. Nevertheless, $D_{1}(t)=-\frac{1}{t(t+1)}$ is not operator monotone. As a matter of fact, we take two $2 \times 2$ matrices $A$ and $B$ :

$$
A=\left(\begin{array}{ll}
3 & 1 \\
1 & 2
\end{array}\right) \quad \text { and } \quad B=\left(\begin{array}{ll}
2 & 0 \\
0 & 1
\end{array}\right) .
$$

Note that $D_{1}(A) \geq D_{1}(B)$ if and only if $A(A+1) \geq B(B+1)$. Clearly $A \geq B$, but

$$
A(A+1)-B(B+1)=\left(\begin{array}{cc}
13 & 6 \\
6 & 7
\end{array}\right)-\left(\begin{array}{ll}
6 & 0 \\
0 & 2
\end{array}\right)=\left(\begin{array}{ll}
7 & 6 \\
6 & 5
\end{array}\right) \nsupseteq 0 .
$$

This is a counterexample.

Incidentally, the operator convexity of the function $\frac{1}{t}$ is easily shown as follows: It is enough to prove the inequality

$$
\left(\frac{A+B}{2}\right)^{-1} \leq \frac{1}{2}\left(A^{-1}+B^{-1}\right) .
$$

And it is simplified by putting $C=A^{\frac{1}{2}} B^{-1} A^{\frac{1}{2}}$ that

$$
4\left(1+C^{-1}\right)^{-1} \leq 1+C,
$$

which follows from the numerical inequality $4 \leq\left(1+x^{-1}\right)(1+x)$.

Acknowledgement. The authors would like to express their hearty thanks to the referee for his/her polite reviewing and kind suggestion.

\section{REFERENCES}

1. J.I. Fujii, M. Fujii and R. Nakamoto, An operator inequality implying the usual and chaotic orders, Ann. Funct. Anal. 5 (2014), 24-25.

2. M. Fujii, T. Furuta and E. Kamei, Furuta's inequality and its application to Ando's Theorem, Linear Algebra Appl. 179 (1993), 161-169.

3. M. Fujii, J.-F. Jiang and E. Kamei, Characterization of chaotic order and its application to Furuta inequality, Proc. Amer. Math. Soc. 125 (1997), 3655-3658.

4. M. Fujii, Furuta inequality and its related topics, Ann. Funct. Anal. 1 (2010), 28-45.

5. T. Furuta, $A \geq B \geq 0$ assures $\left(B^{r} A^{p} B^{r}\right)^{1 / q} \geq B^{(p+2 r) / q}$ for $r \geq 0, p \geq 0, q \geq 1$ with $(1+2 r) q \geq p+2 r$, Proc. Amer. Math. Soc. 101 (1987), 85-88. 
6. T. Furuta, Operator monotone functions, $A>B \geq 0$ and $\log A>\log B$, J. Math. Inequal.7 (2013), 93-96.

7. T. Furuta, Comprehensive survey on an order preserving operator inequality, Banach J. Math. Anal. 7 (2013), 14-40.

8. D.S. Mitrinović, J.E. Pečarić and A.M. Fink, Classical and New Inequalities in Analysis, Kluwer Academic Publishers, Dordrecht/Boston/London, 1993.

9. M.S. Moslehian and H. Najafi, An extension of the Löwner-Heinz inequality, Linear Algebra Appl. 437 (2012), 2359-2365.

10. M. Uchiyama, Strong monotonicity of operator functions, Integral Equations Operator Theory 37 (2000), 95-105.

11. T. Yamazaki, The Riemannian mean and matrix inequalities related to the Ando-Hiai inequality and chaotic order, Oper. Matrices 6 (2012), 577-588.

1 Department of Mathematics, Osaka Kyolku University, Kashiwara, Osaka 582-8582, JAPAN.

E-mail address: mfujii@cc.osaka-kyoiku.ac.jp

2 Department of Mathematics, Suwon University, Bongdamoup, Whasungsi, KYUNGKIDO 445-743, KOREA.

E-mail address: evergreen1317@gmail.com

3 3-4-13, Daihara-cho, Hitachi 316-0021, Japan.

E-mail address: r-naka@net1.jway.ne.jp 